\title{
Insulin Sensitization and Resistance Interrelationship Revisited with a Quantitative Molecular Model Approach
}

\section{Rao M. Uppu ${ }^{1 *}$ and Narasimham L. Parinandi ${ }^{2}$}

${ }^{1}$ Department of Environmental Toxicology and the Health Research Center, Southern University and A \& M College, Baton Rouge, LA 70813, USA ${ }^{2}$ Division of Pulmonary, Allergy, Critical Care, and Sleep Medicine, Dorothy M. Davis Heart and Lung Research Institute, The Ohio State University College of Medicine, Columbus, $\mathrm{OH} 43210$, USA

Glucose is the prime fuel as the source of energy for humans. However, the glucose levels in circulation have to be very tightly maintained. Alterations in the normal glucose levels in blood will lead to abnormal physiological states causing either hypoglycemia (low glucose levels) or hyperglycemia (high glucose levels). When the blood glucose (sugar) levels are higher than $180 \mathrm{mg} / \mathrm{dl}(10 \mathrm{mmol} / \mathrm{l})$, the condition is considered as the hyperglycemic state (diabetes mellitus). Diabetes is a debilitating disorder world-wide and a root cause for several serious complications such as cardiovascular diseases, cerebrovascular diseases, renal disorders, inflammation and immunity, and obesity. Insulin, the protein hormone synthesized by the $\beta$-cells of the pancreas is one of the prime hormones is responsible for the glucose uptake/utilization by the cells in the body and also tightly maintains the homeostasis of the glucose in circulation. There are two states of diabetes: (1) type-1 diabetes and (2) type- 2 diabetes. Type1 diabetes, also called as the insulin-dependent diabetes mellitus (IDDM), manifests due to the autoimmune damage of the $\beta$-cells which then leads to the suppression or cessation of insulin production. Type1 diabetes is also called the "juvenile diabetes". Type- 2 diabetes, also called as the adult-onset diabetes or non-insulin-dependent diabetes mellitus (NIDDM) among humans is caused by either low levels or absence of insulin or insulin resistance (IR). Hyperglycemia caused by low levels or deficiency of insulin can be corrected by the administration of sufficient/required amounts of insulin into the subjects. However, IR which results from the lack of insulin action or ineffective action of insulin (present in physiologically sufficient amounts) is of serious concern and a challenge.

Effective treatments for type-2 diabetes have been seriously sought after world-wide. Early diagnoses of the condition and strategies to combat type-2 diabetic hyperglycemic state have become challenges in the laboratories, clinics, and the society. Alternate strategies such as regimental dietary management, exercise and stress management have been strongly recommended and adopted to control type- 2 diabetes. Among those, diet has been seriously considered in controlling type2 diabetic hyperglycemia. Consumption of refined foods, polished cereals, and fat have been observed to influence the early onset of impaired glucose tolerance (IGT) which eventually leads to diabetes without any prior warning. Treatment of these cases with "insulin sensitizers" such as metformin appears to be promising in alleviating the associated hyperglycemic condition. The present state of understanding of the mechanism of action of metformin hinges on its inhibition of gluconeogenesis in the liver and also insulin sensitization leading to efficient glucose clearance in blood.

The report by Punna, Nappan Veettil, Paruchuri, and Marasanapalle published in this issue of Diabetes and Metabolism entitled "Insulin Sensitization and Resistance Interrelationship in a Prediabetic Rat: A Quantitative Molecular Model", addressed an important aspect of insulin-regulated glucose homeostasis, taking the relationship between insulin sensitization and resistance into serious consideration. The authors elegantly used a pre-diabetic rat model and built a model of interrelationships among insulin and its receptors as both of them regulate the glucose uptake by the tissue and maintain the homeostasis of circulating glucose. The authors provided convincing experimental evidence that the ratio of insulin receptors/insulin molecules and the associated decrease in plasma glucose levels were positively correlated. The novelty of this study was that the authors fed the animals a soluble dietary fiber of fenugreek origin and investigated the modulation of plasma glucose levels in vivo. Plasma glucose levels were lowered by feeding animals the soluble fenugreek fiber but not by insulin treatment by apparently enhancing the interaction of available basal insulin with its receptors and by augmenting their availability on the cell surface of target tissue. The authors described that while the affinity of the receptor to insulin is mole to mole, the availability of the insulin receptors is an absolute requirement due to rheological considerations. It was also deduced that if higher number of receptors encountered per mole of insulin, the glucose lowering effect was greater. On the other hand, the unavailability or inaccessibility of insulin receptors would lead leads to IR leading to lowered glucose uptake by the tissue and poor clearance of blood glucose. The principle of inverse relation between ligand $v s$ receptor dictates the process of glucose uptake by the cells. This is a crucial relationship between insulin and its receptors that oscillates around a fixed position of the receptor on cell membrane as opposed to the notion that the constant availability of insulin molecules arising from $\beta$-cell secretion in response to the glucose load. The collision between insulin and its receptors is dependent upon the formation and the internalization of the ligand-receptor complex. This interrelationship has not been adequately described with experimental evidences to date and this has a significant bearing on the glucose homeostasis in circulation. Thus, the findings of this study offered a novel school of thought that effective interaction of insulin (ligand) with its receptor in the tissue facilitates faster clearance of plasma glucose. In other words, departing from the notion of excessive usage of insulin and focusing more on the insulin receptor may be considered as lowered abundance of the insulin receptors may contribute to IR.

More attractive feature of this study is the use of a computational methodology which is simple and can be done in all laboratories to test this hypothesis of sensitization within a broad framework. At present,

*Corresponding author: Rao M. Uppu, PhD., Department of Environmental Toxicology and the Health Research Center, Southern University and A \& $M$ College, Baton Rouge, LA 70813, USA, Tel: 225-771-4525; Fax: 225-771-5350; E-mail: rao_uppu@subr.edu

Received September 27, 2011; Accepted September 29, 2011; Published September 30, 2011

Citation: Uppu RM, Parinandi NL (2011) Insulin Sensitization and Resistance Interrelationship Revisited with a Quantitative Molecular Model Approach. J Diabetes Metab 2:106e. doi:10.4172/2155-6156.1000106e

Copyright: @ 2011 Uppu RM, et al. This is an open-access article distributed unde the terms of the Creative Commons Attribution License, which permits unrestricted use, distribution, and reproduction in any medium, provided the original author and source are credited. 
Citation: Uppu RM, Parinandi NL (2011) Insulin Sensitization and Resistance Interrelationship Revisited with a Quantitative Molecular Model Approach. J Diabetes Metab 2:106e. doi:10.4172/2155-6156.1000106e

Page 2 of 2

the glycemic indexes of several foods are evaluated for advising diabetic patients. However, the inter- and intra-individual variations are greater and recommendations based on these glycemic indices of foods may have shortcomings. With the aid of concept of insulin sensitization based on the availability of insulin receptors per molecule of insulin, the inter- and intra-individual variation can be overcome for early diagnosis of IR in subjects who are prone for IGT and diabetes which may be a useful prognosis for diabetics.
The inclusion of soluble dietary fiber as a nutraceutical appears to be a beneficial strategy in controlling/combating hyperglycemia associated with type-2 diabetes with IR. More noticeably, the authors put forward an interesting hypothesis stating that there could be certain endogenous factor(s) which could attenuate/inhibit gluconeogenesis and this is similar to endorphins and enkephelins. Nevertheless, extensive research is warranted in this are to identify and isolate these factors and test their biological activities. 